\title{
Toward a multi-sensor neural net approach to automatic text classification
}

\author{
Venu Dasigi $\dagger^{\dagger}$ and Reinhold C. Mann $\dagger$ \\ $\dagger_{\text {Sacred Heart University, Department of Computer Science and }}$ \\ Information Technology, Fairfield, CT 06ł32-1000. e-mail: \\ dasigi@shu.sacredheart.edu \\ $\ddagger_{\text {Oak Ridge National Laboratory, Intelligent Systems Section, Computer }}$ \\ Science and Mathematics Division, Oak Ridge, TN 37831-6364.e-mail: \\ mannrc@ornl.gov
}

\begin{abstract}
Many automatic text indexing and retrieval methods use a term-document matrix that is automatically derived from the text in question. Latent Semantic Indexing, a recent method for approximating large term-document matrices, appears to be quite useful in the problem of text information retrieval, rather than text classification (Deerwester, et al., 90). Here we outline a method that attempts to combine the strength of the LSI method with that of neural networks, in addressing the problem of text classification. In doing so, we also indicate ways to improve performance by adding additional "logical sensors" to the neural network, something that is hard to do with the LSI method when employed by itself. Preliminary results are summarized, but much work remains to be done.
\end{abstract}

\section{Keywords}

Text classification, latent semantic indexing, neural network, logical sensors, reference library, term-document matrix, singular value decomposition 


\section{INTRODUCTION \& BACKGROUND}

Most contemporary approaches to information retrieval use terms contained in a text document directly as indexes into the document. "Vector-based" approaches view documents as vectors of such terms. Thus, a "library" of documents is represented as a term-document matrix, where the entries represent the frequency of each term in each document. Such term-document matrices tend to be very large and sparse.

Latent Semantic Indexing (LSI) is a recent method that captures the "latent semantic structure" of documents, as indicated in a term-document matrix (Deerwester, et al., 90). The large sparse matrix is reduced into three relatively small matrices by singular value decomposition (SVD), whose product approximates the original sparse matrix. Our work is an initial effort to combine the valuable ideas of LSI with the powerful pattern-matching and learning capabilities of neural networks. A major stumbling block in applying neural networks to most IR applications has been that the size of a typical IR problem results in impractically large neural networks. In a sizable application, a document to be classified, as well as a query, is represented as a set of terms, the size of which is in the thousands. The number of connections would be even larger. An LSI-based approach may be used to address the issue.

\section{A NEURAL NET APPROACH WITH MULTIPLE INPUT SENSORS}

Specifically, in this initial effort, we focused on two main goals. First, create input to a neural network that is LSI-based, so that the size of the neural net will be practical. Further, a second goal is to see if additional sensors can be added easily to the neural net input, to give improved results. The relationship between the LSI component and the neural network is symbiotic. The LSI-based input compresses the input to the neural network to a much smaller size. Further, LSI is based on a solid mathematical theory, adding strength to the resulting system. For its part, the neural network adds trainability to the LSI-based method, and also makes it possible to integrate other sensors to supplement the LSI-based input. Initially the system classifies text documents into different categories, and can eventually be modified to retrieve documents that match a specific profile.

A straightforward, but simple-minded input vector for the neural network would be a document represented as a vector of all possible term frequencies, which generally number in the thousands for most sets of documents of reasonable size (such as newspaper or magazine stories, novels, scientific articles, etc.). LSI work suggests a way to represent a document using around a hundred "factors", derived from the much longer term vector and the SVD of a "reference matrix." A reference matrix is the term-document matrix of a reference library/collection of documents. A reference library is simply the collection of documents that "adequately" represents all concepts of interest.

The developers of LSI indicate that a query may be viewed as a pseudo-document and may be represented by a vector of a chosen number of factors (Deerwester, et al., 90). First a reference term-document sparse matrix $X$ is derived from the library of documents that are of interest. This matrix is split into three matrices by SVD, so that 


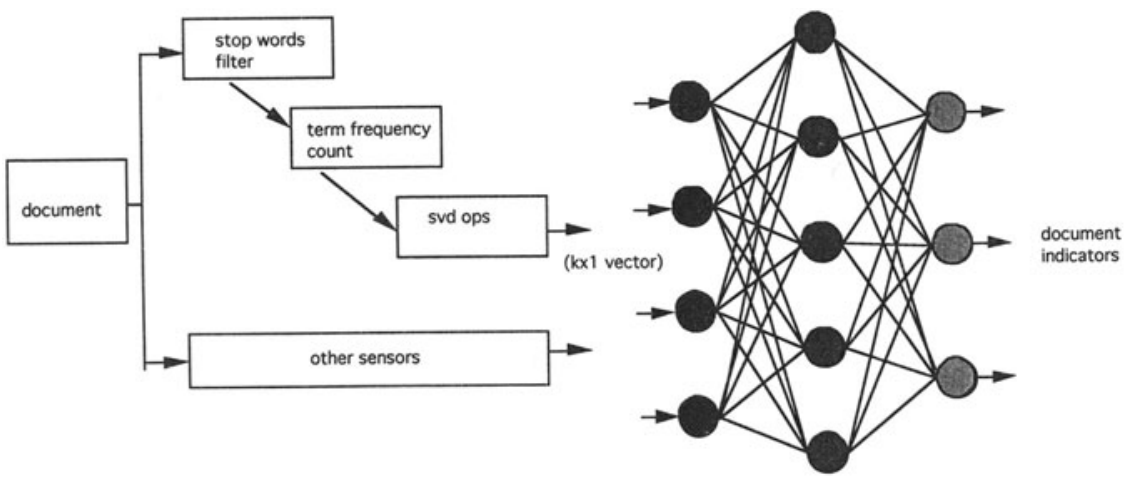

Figure 1 Figure 1. Schematic of the multi-sensor neural network approach to text document classification.

$X=T . S . D^{\prime}$

Here, $X$ is a txd matrix, where $t$ is the numbers of distinct terms (word roots) and $\mathrm{d}$ is the number of documents in the reference collection. The order of $T$ is txk, that of $\mathrm{S}$, which is a diagonal matrix, is kxk, and that of $\mathrm{D}$ is $\mathrm{dxk}$, where $\mathrm{k}$ is the chosen number of factors. Now, the $1 \mathrm{xk}$ pseudo-document vector $D_{Q}$ corresponding to a $1 \mathrm{xt}$ query vector $Q$ may be derived simply as:

$D_{Q}=Q . T . S^{-1}$

In this work, we use this same idea to squash any regular document vector into a $1 \mathrm{xk}$ vector that serves as input to the neural network. The only care that must be exercised is to make sure that the reference term-document matrix that is used as the starting point is one that "adequately" represents all concepts of interest. It may be noted that this requirement is no more stringent than would be required in the standard LSI approach.

Figure 1 shows a high level view of how the proposed method works. The input to the system is an individual document that needs to be classified into one of several categories. Different logical sensors are applied to the document, constituting different kinds of preprocessing to derive salient features. The features derived by the logical sensors constitute input to a neural net that has already been trained. The first such sensor compresses the $1 \mathrm{xt}$ input term vector into a $1 \mathrm{xk}$ vector as explained above. The output is an indication of the category to which the document belongs. A second logical sensor is also used, which is based on simple profiles of the output categories. In this simple sensor, each category profile is simply a set of keywords characterizing that particular category. There is one output per category from this sensor, which represents what fraction of the terms in the given document match the category profile. This second sensor, although rather simple-minded, is intended to help gauge the impact of multiple sensors. 


\section{EXPERIMENTS AND RESULTS}

Our initial focus was on hundreds of AP news wire stories from the TIPSTER collection (Harman, 93). The corpus contains, among other items, AP news wire stories for two full years, tens to hundreds of stories per day. Here, some example output categories are: accidents, crime, business and finance, culture, politics and government, weather, obituary, etc. A major stumbling block in this work was the manual categorization of documents for training and testing purposes. Within the time frame of this initial effort, we could manually categorize only 480 news stories, which is what we worked with. Since the neural networks are of substantial size (see below), they typically require many more training inputs.

Below, we describe the results obtained using two different neural networks, one using just LSI-based inputs and a second one using both LSI-based inputs and those derived from the simple second sensor described above. Of the 480 documents, the first 380 were used as the "reference library". That is the term-document matrix used in all LSI/SVD operations has 380 columns. The number of SVD "factors" used in this work was 112. The neural net inputs were also based on this matrix. The neural net was a simple feedforward net with back propagation, and used the delta rule for learning and the tanh transfer function. It was tested in two configurations: one with 112 inputs (just based on LSI alone) and another with 112 LSI-based inputs plus another 10 inputs based on simple category profiles. Both configurations used 10 output units, one for each category. The neural net architectures that performed best for each configuration are chosen, which happened when the single sensor neural net (with just 112 LSI-based inputs) has 9 hidden units, and the other neural net (with two sensors, for a total of 122 inputs) has 10 hidden units.

We compared the multi-sensor neural net approach against an LSI-based classification. The LSI approach, as described in (Deerwester, et al., 90), was originally intended for document retrieval, rather than classification. The method was modified to perform classification as follows. First the document from the reference library that best matches the input document is identified using the original LSI method. Next the category of this matched reference document is looked up from the training data and is reported as the category for the input document in question. When the LSI method was used by itself to classify the 100 documents that are outside the reference library, the results were somewhat surprising. LSI classified the reference library documents with a perfect $100 \%$ accuracy, the percentage of correct results when the 100 new documents were used dropped to $54 \%$. Only one such experiment was conducted because generating the SVD for each new reference library was computationally very expensive, even when several megabytes of main memory was used by the program.

We present the results of our neural network experiments in two tables. The percentages of correct results shown in the tables represent the peak performance that did not get any better with more iterations. For testing the neural nets, inputs were created for all the 480 documents, including a correct answer for each case. This "answer" was to be used either for training the neural net or for comparison, in the case of a testing. The data were cross validated by generating a dozen pairs of training and test inputs with an approximate $90 \%-10 \%$ split (that is 430 training inputs and 50 test inputs). The same pairs of data sets were used to test both the single sensor neural net and the two sensor one. 
Table 1 Results of Classification with the Single Sensor Neural Net

\begin{tabular}{llll}
\hline $\begin{array}{l}\text { Data Set } \\
\text { No. }\end{array}$ & $\begin{array}{l}\text { No. of } \\
\text { Iterations }\end{array}$ & $\begin{array}{l}\text { Percent Correct } \\
\text { with Test Data }\end{array}$ & $\begin{array}{l}\text { Percent Correct } \\
\text { with Training Data }\end{array}$ \\
\hline 1 & $48 \mathrm{~K}$ & 58 & 80.70 \\
2 & $16 \mathrm{~K}$ & 72 & 77.67 \\
3 & $64 \mathrm{~K}$ & 72 & 76.98 \\
4 & $64 \mathrm{~K}$ & 62 & 77.21 \\
5 & $32 \mathrm{~K} \cdot$ & 62 & 76.05 \\
6 & $48 \mathrm{~K}$ & 62 & 76.98 \\
7 & $80 \mathrm{~K}$ & 62 & 80.47 \\
8 & $48 \mathrm{~K}$ & 58 & 78.14 \\
9 & $64 \mathrm{~K}$ & 68 & 77.44 \\
10 & $48 \mathrm{~K}$ & 60 & 78.14 \\
11 & $48 \mathrm{~K}$ & 64 & 78.84 \\
12 & $32 \mathrm{~K}$ & 68 & 76.05 \\
\hline
\end{tabular}

Table 2 Results of Classification with the Two Sensor Neural Net

\begin{tabular}{llll}
\hline $\begin{array}{l}\text { Data Set } \\
\text { No. }\end{array}$ & $\begin{array}{l}\text { No. of } \\
\text { Iterations }\end{array}$ & $\begin{array}{l}\text { Percent Correct } \\
\text { with Test Data }\end{array}$ & $\begin{array}{l}\text { Percent Correct } \\
\text { with Training Data }\end{array}$ \\
\hline 1 & $48 \mathrm{~K}$ & 62 & 85.11 \\
2 & $48 \mathrm{~K}$ & 70 & 84.42 \\
3 & $32 \mathrm{~K}$ & 76 & 84.19 \\
4 & $64 \mathrm{~K}$ & 64 & 83.72 \\
5 & $32 \mathrm{~K}$ & 64 & 84.88 \\
6 & $16 \mathrm{~K}$ & 66 & 83.26 \\
7 & $32 \mathrm{~K}$ & 66 & 84.88 \\
8 & $16 \mathrm{~K}$ & 62 & 84.19 \\
9 & $32 \mathrm{~K}$ & 70 & 83.49 \\
10 & $32 \mathrm{~K}$ & 58 & 83.02 \\
11 & $16 \mathrm{~K}$ & 64 & 80.47 \\
12 & $32 \mathrm{~K}$ & 72 & 83.02 \\
\hline
\end{tabular}

Table 1 shows the performance of the 112-input neural net on each of the dozen different data sets. In each set, the test file was tested after the neural net was trained for anywhere between 16,000 and 64,000 iterations. On the test set, the correctness percentage ranged from a minimum of $58 \%$ to a maximum of $72 \%$ for the dozen sets. When the training set itself was used as a data set, the performance was between $76.05 \%$ to $80.7 \%$ correct.

Table 2 shows the performance of the 122-input neural net on each of the dozen different data sets. Again, in each set, the test file was tested after the neural net was trained for anywhere between 16,000 and 64,000 iterations. On the test set, the correctness percentage ranged from a minimum of $58 \%$ to a maximum of $76 \%$ for the dozen sets. When the training set itself was used as a data set, the performance was between $80.47 \%$ to $85.11 \%$ correct. It may be seen that in the case of individual data sets, there was an improvement in performance with 10 out of the 12 data sets in the two sensor neural net, compared to 
the single sensor version, with marginal decrease of performance in the other two cases. But in one of these two cases (data set 2), the superficially better performance of the single sensor neural net decreased a few percent with more training. These anomalies can perhaps be attributed to the simple-mindedness of the second sensor that was employed.

With the neural nets, although the need for more training inputs is obvious, there is a clear improvement of classification results compared to the LSI method by itself. And the two sensor version, even with its simple-minded second sensor, performs better in most cases than the single sensor version. Other researchers appear to have evaluated LSIbased approaches, too (Schütze, Hull and Pederson, 95). Our approach differs from theirs in using a reference library, and in employing multiple sensors. In their work, they seem to have used the set of all tested documents together as the reference library, obviating the need for a hidden layer in the neural network. We believe this approach is not practical. It may be noted that SVD is computationally very expensive, but our approach performs an SVD just once - on the reference collection. As already pointed out, the choice of the reference library can be critical.

Ongoing and future work plans include further training of the nets, developing other more informative sensors, possibly using natural language techniques (for faster training), strengthening the category profiles in the second sensor by various means, etc.

\section{ACKNOWLEDGEMENTS}

Venu Dasigi thanks the Oak Ridge Institute for Science and Education for the fellowship that made this work possible. Both authors thank Mike Berry for the SVDPACKC software.

\section{REFERENCES}

[Deerwester, et al., 90] Deerwester, S., S. Dumais, G. Furnas, T. Landauer and R. Harshman, Indexing by Latent Semantic Analysis Journal of the American Society for Information Science, 41(6), pp. 391-407.

[Harman, 93] Harman, D., Overview of the First TREC Conference, Proc. of SIGIR-93, pp. 391-407.

[Schütze, Hull and Pederson, 95] Schütze, H., D. Hull and J, Pedersen, A Comparison of Classifiers and Document Representations for the Routing Problem, Proc. of SIGIR-95, pp. 229-37.

\section{BIOGRAPHY}

Venu Dasigi is Associate Professor and Graduate Program Director at Sacred Heart University. He received a Ph.D. in Computer Science from the University of Maryland, College Park. Previously, he was a Computer Science faculty member at Wright State University and also worked as a Development Engineer for Philips India. He received a U.S. Department of Energy Summer Research Fellowship and worked at the Oak Ridge National 
Laboratory in 1995 and 1996. He organized a AAAI-91 workshop on abductive inference. He published over twenty papers in AI and Computer Science. He is a member of ACM, IEEE-CS, AAAI and ASIS.

Reinhold C. Mann received a Diplom-Mathematiker degree in 1977, and a Dr. rer. nat. degree in physics in 1980 from the Johannes Gutenberg University in Mainz, Germany. He held appointments as consultant and research staff member at the University in Mainz, the Battelle Institute in Frankfurt and at the Fraunhofer Institute for Data and Information Processing in Karlsruhe, Germany, before coming to the USA in 1981 as an Alexander von Humboldt Foundation Feodor-Lynen Fellow at Oak Ridge National Laboratory (ORNL). He joined the staff at ORNL in 1983 and has been head of the Intelligent Systems Section since 1989 . 